\title{
Relic gravitational wave spectrum, the trans-Planckian physics and Hořava-Lifshitz gravity
}

\author{
Seoktae Koh* \\ Institute of Theoretical Physics, Chinese Academy of Sciences, \\ P.O. Box 2735, Beijing, 100080, China
}

\begin{abstract}
We calculate the spectrum of the relic gravitational wave due to the trans-Planckian effect in which the standard linear dispersion relations may be modified. Of the modified dispersion relations suggested in literatures which have investigated the trans-Planckian effect, we especially use the Corley-Jacobson dispersion relations. The Corley-Jacobson type modified dispersion relations can be obtained from Hořava-Lifshitz gravity which is non-relativistic and UV complete. Although it is not clear how the transitions from Hořava-Lifshitz gravity in the UV regime to Einstein gravity in the IR limit occur, we assume Hořava-Lifshitz gravity regime is followed by the inflationary phase in Einstein gravity.
\end{abstract}

*email: skoh@itp.ac.cn 


\section{INTRODUCTION}

In spite of the success of the inflationary scenario, inflation is still encountered several unsolved problems. Among them, the trans-Planckian problem [1] is related to the fundamental problem of the high energy physics i.e quantum theory of gravity. Unfortunately there is no successful quantum gravity theory for handling the spacetime around the Planck scale, so one way out of such difficulties is to use the effective modified dispersion relations to mimic the effect of the trans-Planckian physics. Of the several modified dispersion relations suggested in many literatures, we will use the Corley-Jacobson dispersion relation [1] [2] in this work.

The Corley-Jacobson type dispersion relations could be derived naturally from the recently proposed quantum gravity model called Hořava-Lifshitz(HL) gravity [3] [4]. HL gravity is a non-relativistic and renormalizable theory in the UV region. In addition, because of the different scaling behaviors of the time and space $\left(t \rightarrow l^{3} t, \mathbf{x} \rightarrow l \mathbf{x}\right)$, while the kinetic term is still quadratic order in time derivatives of the metric in the action, the potential terms may have up to 6th order spatial derivatives [5] [6]. HL gravity may also provide the seeds for the large scale structure without inflation [6] [7]. On the other hand, it is still controversial if HL gravity is a complete theory [8] which would be dependent upon the projectability and the detail balance condition [3]. No explicit inconsistencies has been pointed out against the version without the detailed balance condition with the projectability condition [9]. And it is also not clear how the transition from HL gravity in the UV regime to Einstein gravity in the IR limit takes place.

In this paper, we calculate the spectrum of the relic gravitational wave in order to investigate the effect of the trans-Planckian physics. Especially, we use the Corley-Jacobson dispersion relations taking HL gravity into account. For this purpose, we assume the HL gravity phase is followed by the inflationary phase in Einstein gravity. Though the transition time from the trans-Planckian regime to the usual standard inflationary period is usually determined by the cut-off momentum, we will leave it as a free parameter assuming it keep the information about the transition mechanism. The resulting spectrum could be constrained from the experimental bounds such as the ground based gravitational wave detector (LIGO, VIRGO) which covers the frequency range $10 \mathrm{~Hz}<f<10^{3} \mathrm{~Hz}$ and future planned space based one (LISA) which is expected to cover $10^{-3} \mathrm{~Hz}<f<1 \mathrm{~Hz}$ or the cosmological bounds 
(nucleosynthesis, CMB) in the low frequency range [10].

The paper is organized as follows. In Sect. [II, we calculate the power spectrum of the tensor perturbations with the Corley-Jacobson dispersion relations. In Sect. III, we obtain the equation for the tensor perturbations in HL gravity and calculate the power spectrum. In Sect. IV, we investigate the effect of the trans-Planckian physics and HL gravity on the spectrum of the relic gravitational wave. And finally, we summarize in Sect. V.

\section{GRAVITATIONAL WAVE SPECTRUM IN THE TRANS-PLANCKIAN PHYSICS}

We briefly review the effect of the trans-Planckian physics on the power spectrum in this section. With the metric

$$
d s^{2}=a^{2}(\eta)\left[-d \eta^{2}+\left(\delta_{i j}+h_{i j}\right) d x^{i} d x^{j}\right]
$$

where $h_{i j}$ is a transverse, traceless perturbation which can be expanded as

$$
h_{i j}(x)=\frac{1}{a} \int \frac{d^{3} k}{(2 \pi)^{3 / 2}} \sum_{i j} \epsilon_{i j} u_{k}(\eta) e^{i \mathbf{k} \cdot \mathbf{x}},
$$

and $\epsilon_{i j}$ is a polarization tensor, the tensor perturbations are governed by the equations

$$
u_{k}^{\prime \prime}+\left(k^{2}-\frac{a^{\prime \prime}}{a}\right) u_{k}=0
$$

where a prime denotes a derivative with respect to the conformal time and $u_{k}$ satisfy the normalization condition

$$
u_{k} u_{k}^{* \prime}-u_{k}^{*} u_{k}^{\prime}=i
$$

This equation is believed to hold only at the low energy scale, $k_{\text {phys }} \ll M_{p l}$, where $k_{\text {phys }}=$ $k / a$. As we go back in time around the Planck scale, quantum effects would be important and it seems to be necessary introducing the quantum gravity theory in order to describe correctly the Planck scale phenomena. Unfortunately, however, no successful quantum gravity theory exist at present. Though the string theory is believed to be a strong candidate of the quantum gravity theory, it is not complete yet. Thus, one way to overcome these difficulties and to mimic the quantum gravity effects around the Planck scale is to consider the modified dispersion relations. 
Taking into account of the modified dispersion relations, $\omega(k / a)$, the equation for $u_{k}$ could have the following form

$$
u_{k}^{\prime \prime}+\left(\omega^{2}(k / a)-\frac{a^{\prime \prime}}{a}\right) u_{k}=0 .
$$

In this paper, we take the Corley-Jacobson dispersion relations [2] [1] as an example of the modified dispersion relations

$$
\omega^{2}(k / a)=k^{2}\left(1+b_{m}\left(\frac{k}{a M}\right)^{2 m}\right),
$$

where $M$ is a cutoff scale and $b_{m}$ is an arbitrary coefficient. Here $k$ is a comoving wave number. Depending on the sign of $b_{m}$ which can be positive or negative [1], we may reach to the different results. If $b_{m}<0, \omega^{2}$ becomes a complex function for $k>\left|b_{m}\right|^{-1 / 2 m} a M$ and the WKB approximation may not be valid [11] in this region. For the purpose of this work, bearing in mind HL gravity, it is enough to limit to the positive $b_{m}$. From now on we only consider $b_{m}>0$. (For $b_{m}<0$, see Ref. [1] ).

If we assume exponential inflation

$$
a(\eta)=-\frac{1}{H \eta}
$$

the equation (5) yields

$$
u_{k}^{\prime \prime}+\left(k^{2}+\sigma^{2}(k) k^{2 m+2} \eta^{2 m}-\frac{2}{\eta^{2}}\right) u_{k}=0,
$$

where

$$
\sigma^{2}(k)=b_{m}\left(\frac{H}{M}\right)^{2 m} .
$$

For $k>a M$, since $\omega^{2}(k / a) \approx \sigma^{2} k^{2 m+2} \eta^{2 m}$, the equation (8) becomes

$$
u_{k}^{\prime \prime}+\left(\sigma^{2} k^{2 m+2} \eta^{2 m}-\frac{2}{\eta^{2}}\right) u_{k}=0
$$

and its solutions are given by

$$
u_{k}=c_{1} \sqrt{\frac{\pi}{4(1+m)}}|\eta|^{1 / 2} H_{\nu}^{(1)}(z)+c_{2} \sqrt{\frac{\pi}{4(1+m)}}|\eta|^{1 / 2} H_{\nu}^{(2)}(z),
$$

where $H_{\nu}^{(1)}$ and $H_{\nu}^{(2)}$ are the Hankel function of the first and second kind with the order $\nu$, respectively, and

$$
z=\frac{\sigma}{1+m}(k \eta)^{1+m}, \quad \nu=\frac{3}{2(1+m)}
$$


For $b_{m}<0$, the solutions are given by the modified Bessel functions.

If we choose the positive frequency modes as initial conditions when $k>a M$, we can choose $c_{1}=0, c_{2}=1$ taking the normalization condition (44) into account. This choice of the initial vacuum is equivalent to the minimizing the energy density[1] up to the phase factor

$$
u_{k}\left(\eta_{i}\right)=\frac{1}{\sqrt{2 \omega}}, \quad u_{k}^{\prime}\left(\eta_{i}\right)=-i \sqrt{\frac{\omega}{2}},
$$

if the adiabatic approximation $\left(\left|\omega^{\prime}\right| \ll \omega^{2}\right)$ is hold. Then the mode solutions of (10) for $k>a M$ can be written as

$$
u_{k}=\sqrt{\frac{\pi}{4(1+m)}}|\eta|^{1 / 2} H_{\nu}^{(2)}(z) .
$$

For $k<a M$, the dispersion relations recover the standard linear relations, $\omega^{2} \simeq k^{2}$, as in (3) and then the mode solutions are given by

$$
u_{k}(\eta)=\frac{\mathcal{A}_{k}}{\sqrt{2 k}}\left(1-\frac{i}{k \eta}\right) e^{-i k \eta}+\frac{\mathcal{B}_{k}}{\sqrt{2 k}}\left(1+\frac{i}{k \eta}\right) e^{i k \eta},
$$

where the coefficients satisfy the normalization condition

$$
\left|\mathcal{A}_{k}\right|^{2}-\left|\mathcal{B}_{k}\right|^{2}=1
$$

The coefficients are determined through the matching conditions at $\eta=\eta_{1}$ where $\eta_{1}$ is the time when the modified dispersion relations take the standard linear form,

$$
k\left|\eta_{1}\right|=\frac{1}{b_{m}^{1 / 2 m}} \frac{M}{H} \gg 1 .
$$

Through the matching conditions at $\eta=\eta_{1}$, we obtain the coefficients

$$
\begin{aligned}
\mathcal{A}_{k}= & \sqrt{\frac{\pi}{8(1+m)}}\left(k\left|\eta_{1}\right|\right)^{1 / 2}\left(1+\frac{3 i}{2 k\left|\eta_{1}\right|}-\frac{3}{2 k^{2}\left|\eta_{1}\right|^{2}}\right) e^{i k\left|\eta_{1}\right|} H_{\nu}^{(2)}\left(z_{1}\right) \\
& +\sqrt{\frac{\pi(1+m)}{8}} \frac{i z_{1}}{\left(k\left|\eta_{1}\right|\right)^{1 / 2}}\left(1+\frac{i}{k\left|\eta_{1}\right|}\right) e^{i k\left|\eta_{1}\right|} \frac{d}{d z} H_{\nu}^{(2)}\left(z_{1}\right), \\
\mathcal{B}_{k}= & \sqrt{\frac{\pi}{8(1+m)}}\left(k\left|\eta_{1}\right|\right)^{1 / 2}\left(1-\frac{3 i}{2 k\left|\eta_{1}\right|}-\frac{3}{2 k^{2}\left|\eta_{1}\right|^{2}}\right) e^{-i k\left|\eta_{1}\right|} H_{\nu}^{(2)}\left(z_{1}\right) \\
& -\sqrt{\frac{\pi(1+m)}{8}} \frac{i z_{1}}{\left(k\left|\eta_{1}\right|\right)^{1 / 2}}\left(1-\frac{i}{k\left|\eta_{1}\right|}\right) e^{-i k\left|\eta_{1}\right|} \frac{d}{d z} H_{\nu}^{(2)}\left(z_{1}\right),
\end{aligned}
$$

where

$$
z_{1}=\frac{\sigma}{(1+m)}\left(k\left|\eta_{1}\right|\right)^{1+m}
$$


With these coefficients, we can compute the power spectrum of the gravitational wave at $\eta \rightarrow 0$ or $k \eta \ll 1$

$$
\begin{aligned}
\mathcal{P}_{h} & =\frac{1}{a^{2}} \mathcal{P}_{u}=\frac{k^{3}}{2 \pi^{2}} \frac{\left|u_{k}\right|^{2}}{a^{2}} \\
& =\left(\frac{H}{2 \pi}\right)^{2}\left(1+2\left|\mathcal{B}_{k}\right|^{2}-\operatorname{Re}\left(\mathcal{A}_{k} \mathcal{B}_{k}^{*}\right)\right),
\end{aligned}
$$

where we have used $a=-\frac{1}{H \eta}$ at the second line.

For $k\left|\eta_{1}\right| \gg 1$, using (17) and (20), we finally obtain

$$
\mathcal{P}_{h}(k) \approx\left(\frac{H}{2 \pi}\right)\left(2-\frac{b_{m}^{1 / 2 m}}{4} \frac{H}{M} \sin \left(2 k\left|\eta_{1}\right|\right)\right),
$$

where we have used the asymptotic form of the Hankel function

$$
H_{\nu}^{(2)}(z) \simeq \sqrt{\frac{2}{\pi z}} e^{-i\left(z-\left(\nu+\frac{1}{2}\right) \frac{\pi}{2}\right)}, \quad \text { if } z \gg 1,
$$

and

$$
\frac{d}{d z} H_{\nu}=-H_{\nu+1}+\frac{\nu}{z} H_{\nu}
$$

The power spectrum (22) is the well-known result of the trans-Planckian effect. The leading correction term due to the modified dispersion relations leads to $\frac{H}{M}$. The detectability of this effect is an important concern at the future experiments.

\section{GRAVITATIONAL WAVE IN HOŘAVA-LIFSHITZ GRAVITY}

The quadratic action of Hořava-Lifshitz gravity with the metric (1) is

$$
\begin{aligned}
\delta_{2} \mathcal{S}_{T}= & \int d t d^{3} x a^{2}\left[\frac{2}{\kappa^{2}} h_{i j}^{\prime} h^{i j \prime}+\alpha_{1} h^{i j} \triangle h_{i j}+\frac{\alpha_{2}}{a^{2}} \triangle h^{i j} \triangle h_{i j}\right. \\
& \left.+\alpha_{3} \frac{\epsilon^{i j k}}{a^{3}} \triangle h_{i l} \nabla_{j} \triangle h_{k}^{l}-\frac{\alpha_{4}}{a^{4}} \triangle h^{i j} \triangle^{2} h_{i j}\right],
\end{aligned}
$$

where $\nabla_{i}$ is a covariant derivative with respect to $\delta_{i j}$ and $\triangle=\delta^{i j} \nabla_{i} \nabla_{j}$. The coefficients in the action corresponding to the original theory [3] are

$$
\alpha_{1}=\frac{\kappa^{2} \mu^{2}}{8(1-3 \lambda)} \Lambda, \quad \alpha_{2}=-\frac{\kappa^{2} \mu^{2}}{8}, \quad \alpha_{3}=\frac{\kappa^{2} \mu}{2 w^{2}}, \quad \alpha_{4}=-\frac{\kappa^{2}}{2 w^{4}},
$$

where $\kappa, \lambda, w$ and $\mu$ are coupling constants with scaling dimensions $[\kappa]=[w]=[\lambda]=0,[\mu]=$ 1 and $\Lambda$ is the 3 -dimensional cosmological constant with scaling dimension $[\Lambda]=2$. Einstein gravity is recovered if $\lambda$ is approaching unity in the IR limit. 
The background Friedmann equation in HL gravity [3] is given by [5]

$$
H^{2} \equiv \frac{\dot{a}^{2}}{a^{2}}=\frac{\kappa^{2}}{6(3 \lambda-1)} a^{2}(\rho-\sigma),
$$

where $\rho$ is the background energy density of the matter and

$$
\sigma=-\frac{\kappa^{2} \mu^{2}}{8(1-3 \lambda)} 3 \Lambda^{2}
$$

If $\rho=0$, there is no cosmological solution [5] [12]. In order to get de Sitter solutions, we need to introduce the matter where we assume the vacuum energy density of the matter is dominant. In what follows, we assume $\rho_{0} \equiv \Lambda_{m}>\sigma$, and then the scale factor is given by

$$
a=-\frac{1}{H \eta} \text {. }
$$

Note that by the analytic continuation of the parameters $\mu \rightarrow i \mu$ and $w^{2} \rightarrow i w^{2}$ [13], the de Sitter solutions can be obtained without introducing the matter [12]. The sign of the coefficients in the transformed action is also changed. In order to couple matter with gravity consistently, the relativistic formulation of HL gravity are also investigated [14], in which, although being fully-relativistic, it results to be locally anisotropic in the time-like and space-like directions defined by a family of irrotational observers.

In the action (24), because of $\alpha_{3}$ term, the tensor perturbations are not invariant under the parity transformation $(\mathbf{x} \rightarrow-\mathbf{x})$ [15]. The chirality of the gravitational wave seems to give rise to the important effect for discriminating HL gravity from the other quantum gravity theories at the future experiment [15] [16]. But since the $\alpha_{3}$ term is subdominant compared to the $\alpha_{4}$ term in the UV limit, we will drop such term in the present work.

Varying the action (24) leads to the equation for the tensor perturbations in HL gravity

$$
u_{k}^{\prime \prime}+\left[\alpha_{1} \kappa^{2} k^{2}\left(1+\frac{\left|\alpha_{2}\right|}{\alpha_{1} a^{2}} k^{2}+\frac{\left|\alpha_{4}\right|}{\alpha_{1} a^{4}} k^{4}\right)-\frac{2}{\eta}\right] u_{k}=0,
$$

where we have used the mode expansion (2). In the UV limit, the dispersion relation of this equation is exactly equivalent to the Corley-Jacobson dispersion relation if $m=2$. If only the dominant term is kept with putting

$$
M^{4}=\frac{1}{\kappa^{2}\left|\alpha_{4}\right|}, \quad b_{m}=1,
$$

then the positive mode solution is

$$
u_{k}=\sqrt{\frac{\pi}{12}}|\eta|^{1 / 2} H_{1 / 2}^{(2)}(z), \quad z=\left(\frac{H}{\sqrt{3} M}\right)^{2}(k \eta)^{3} .
$$


Since the Hankel function with the order $\nu=1 / 2$ can be expressed as

$$
H_{1 / 2}^{(2)}(z)=i \sqrt{\frac{2}{\pi z}} e^{-i z},
$$

the power spectrum of the tensor perturbations in HL gravity

$$
\mathcal{P}_{u}(k) \propto k^{3}\left|u_{k}\right|^{2} \propto k^{0}
$$

can be scale invariant in the UV region. If HL gravity can provide the seeds for the large scale structure without the inflationary phase as was suggested in [6] [7], it would also be interesting to find some observational signature due to the tensor perturbations in HL gravity without introducing the inflationary phase [17]. But in this work, we assume HL gravity in the UV region is followed by the inflationary phase in Einstein gravity.

We, then, move to the IR limit. Since it is expected Einstein gravity would be recovered in the IR limit with $\lambda=1$, the equation for the tensor perturbations takes the standard form as like in(3). The solutions, then, are

$$
u_{k}=\frac{\mathcal{A}_{k}}{\sqrt{2 k}}\left(1-\frac{i}{k \eta}\right) e^{-i k \eta}+\frac{\mathcal{B}_{k}}{\sqrt{2 k}}\left(1+\frac{i}{k \eta}\right) e^{i k \eta} .
$$

As in Sect. II, we use the matching conditions at $\eta=\eta_{1}$ in order to determine the coefficients $\mathcal{A}_{k}$ and $\mathcal{B}_{k}$. Although it is unclear how the transition from HL gravity in the UV region to Einstein gravity in the IR limit occurs, we assume that the transition occurs instantaneously at $\eta=\eta_{1}$.

We, then, obtain the coefficients via the matching conditions

$$
\begin{aligned}
\mathcal{A}_{k}= & \sqrt{\frac{\pi}{24}}\left(k\left|\eta_{1}\right|\right)^{1 / 2}\left(1+\frac{3 i}{2 k\left|\eta_{1}\right|}-\frac{3}{2 k^{2}\left|\eta_{1}\right|^{2}}\right) e^{i k\left|\eta_{1}\right|} H_{1 / 2}^{(2)}\left(z_{1}\right) \\
& +\sqrt{\frac{3 \pi}{8}} \frac{i z_{1}}{\left(k\left|\eta_{1}\right|\right)^{1 / 2}}\left(1+\frac{i}{k\left|\eta_{1}\right|}\right) e^{i k\left|\eta_{1}\right|} \frac{d}{d z} H_{1 / 2}^{(2)}\left(z_{1}\right), \\
\mathcal{B}_{k}= & \sqrt{\frac{\pi}{24}}\left(k\left|\eta_{1}\right|\right)^{1 / 2}\left(1-\frac{3 i}{2 k\left|\eta_{1}\right|}-\frac{3}{2 k^{2}\left|\eta_{1}\right|^{2}}\right) e^{-i k\left|\eta_{1}\right|} H_{1 / 2}^{(2)}\left(z_{1}\right) \\
& -\sqrt{\frac{3 \pi}{8}} \frac{i z_{1}}{\left(k\left|\eta_{1}\right|\right)^{1 / 2}}\left(1-\frac{i}{k\left|\eta_{1}\right|}\right) e^{-i k\left|\eta_{1}\right|} \frac{d}{d z} H_{1 / 2}^{(2)}\left(z_{1}\right),
\end{aligned}
$$

where

$$
z_{1}=\left(\frac{H}{\sqrt{3} M}\right)^{2}\left(k\left|\eta_{1}\right|\right)^{3} .
$$


Since, for $k \eta \ll 1$,

$$
\begin{aligned}
\left|\mathcal{B}_{k}\right|^{2} & =\frac{1}{4 k^{2}\left|\eta_{1}\right|^{2}} \\
\operatorname{Re}\left(\mathcal{A}_{k} \mathcal{B}_{k}^{*}\right) & =\frac{1}{4}\left[\frac{1}{k^{2}\left|\eta_{1}\right|^{2}} \cos \left(2 k\left|\eta_{1}\right|\right)+\frac{2}{k\left|\eta_{1}\right|} \sin \left(2 k \mid \eta_{1}\right)\right],
\end{aligned}
$$

the power spectrum of the gravitational wave in the late time is, from (21),

$$
\mathcal{P}_{h}(k) \approx\left(\frac{H}{2 \pi}\right)\left(1-\frac{H}{2 M} \sin \left(\frac{2 M}{H}\right)\right)
$$

where, similarly as in (17), we have used

$$
\left|\eta_{1}\right|=\frac{M}{H k} .
$$

Although we use the relation (41) in order to get (40), since we do not have any information about the transition mechanism, we will, for the time being, leave $\eta_{1}$ as a free parameter which is assumed to keep the information about the transition.

Then, for $k\left|\eta_{1}\right| \gg \frac{M}{H}$, using

$$
\begin{aligned}
\left|\mathcal{B}_{k}\right|^{2} & \approx \frac{H^{2}}{4 M^{2}} k^{2}\left|\eta_{1}\right|^{2} \\
\operatorname{Re}\left(\mathcal{A}_{k} \mathcal{B}_{k}^{*}\right) & \approx-\frac{H^{2}}{4 M^{2}} k^{2}\left|\eta_{1}\right|^{2} \cos \left(2 k\left|\eta_{1}\right|\right),
\end{aligned}
$$

we obtain the power spectrum at the late time

$$
\mathcal{P}_{h}(k) \approx\left(\frac{H}{2 \pi}\right)^{2} \frac{H^{2}}{4 M^{2}} k^{2}\left|\eta_{1}\right|^{2}\left(2+\cos \left(2 k\left|\eta_{1}\right|\right)\right) .
$$

If we assume $\eta_{1} \propto k^{-n}$, the resulting power spectrum behaves $\mathcal{P}_{n} \propto k^{2-2 n}$. In order to fit to the observations, $n \approx 1$.

For $k\left|\eta_{1}\right| \ll \frac{M}{H}$,

$$
\begin{aligned}
\left|\mathcal{B}_{k}\right|^{2} & \approx \frac{M^{2}}{4 H^{2}} \frac{1}{k^{2}\left|\eta_{1}\right|^{2}} \\
\operatorname{Re}\left(\mathcal{A}_{k} \mathcal{B}_{k}^{*}\right) & \approx \frac{M^{2}}{4 H^{2}} \frac{1}{k^{2}\left|\eta_{1}\right|^{2}} \cos \left(2 k\left|\eta_{1}\right|\right),
\end{aligned}
$$

then the power spectrum is

$$
\mathcal{P}_{h}(k) \approx\left(\frac{H}{2 \pi}\right)^{2} \frac{M^{2}}{4 H^{2}} k^{-2}\left|\eta_{1}\right|^{-2}\left(2-\cos \left(2 k\left|\eta_{1}\right|\right)\right) .
$$

Similarly for $k n\left|\eta_{1}\right| \gg M / H$, if we assume $\eta_{1} \propto k^{-n}, \mathcal{P}_{h} \propto k^{-2+2 n}$ and it is required to be $n \approx 1$ in order for the spectrum to be scale invariant. Otherwise, it may not satisfy the observations. $M / H$ can also be constrained from the observations. 


\section{SPECTRUM OF THE RELIC GRAVITATIONAL WAVE}

In this section, we compute the number of gravitons produced at the de Sitter- radiation dominated phase transition in order to estimate the intensity of the gravitational wave due to the effect from the trans-Planckian physics for the detection at the gravitational wave detectors.

With the scale factor during the radiation dominated phase $\left(\eta_{e}<\eta<\eta_{e q}\right)$

$$
a(\eta)=\frac{1}{H \eta_{e}^{2}}\left(\eta-2 \eta_{e}\right)
$$

where $\eta_{e}$ is the time when inflation ends, the mode solutions of (3) are given by

$$
u_{k}(\eta)=\frac{\alpha_{k}}{\sqrt{2 k}} e^{-i k \eta}+\frac{\beta_{k}}{\sqrt{2 k}} e^{i k \eta}
$$

and the coefficients are satisfied the normalization condition

$$
\left|\alpha_{k}\right|^{2}-\left|\beta_{k}\right|^{2}=1
$$

Requiring the mode solutions (49) during the radiation dominated phase and (15) during the inflationary period to be continuous at $\eta=\eta_{e}$ gives

$$
\begin{aligned}
\alpha_{k} & =\mathcal{A}_{k}\left(1-\frac{i}{k \eta_{e}}-\frac{1}{2 k^{2} \eta_{e}^{2}}\right)+\mathcal{B}_{k} \frac{1}{2 k^{2} \eta_{e}^{2}} e^{2 i k \eta_{e}} \\
\beta_{k} & =\mathcal{A}_{k} \frac{1}{2 k^{2} \eta_{e}^{2}} e^{-2 i k \eta_{e}}+\mathcal{B}_{k}\left(1+\frac{i}{k \eta_{e}}-\frac{1}{k^{2} \eta_{e}^{2}}\right)
\end{aligned}
$$

The spectrum of the relic gravitational waves is expressed in terms of the number of gravitons produced at the transition [10]

$$
\Omega_{g w}(f)=\frac{1}{\rho_{c}} \frac{d \rho_{g w}}{d \log f}=\frac{16 \pi^{2} f^{4}}{\rho_{c}} N_{k},
$$

where $\rho_{g w}$ is the energy density of the stochastic background gravitational waves, $f$ is the physical frequency and $\rho_{c}=3 H_{0}^{2} M_{P}^{2} /(8 \pi)$ is the critical energy density today.

From (52), $N_{k}$ is given by

$$
\begin{array}{r}
N_{f}=\left|\beta_{k}\right|^{2}=\frac{1}{4 k^{4} \eta_{e}^{4}}+\left|\mathcal{B}_{k}\right|^{2}\left(1-\frac{1}{k^{2} \eta_{e}^{2}}+\frac{5}{4 k^{4} \eta_{e}^{4}}\right) \\
+\frac{1}{k^{2} \eta_{e}^{2}} \operatorname{Re}\left[e^{-2 i k \eta_{e}} \mathcal{A}_{k} \mathcal{B}_{k}^{*}\left(1-\frac{i}{k \eta_{e}}-\frac{1}{k^{2} \eta_{e}^{2}}\right)\right]
\end{array}
$$


where we have used $\left|\mathcal{A}_{k}\right|^{2}-\left|\mathcal{B}_{k}\right|^{2}=1$. Since $k_{\text {phys }}=\frac{k}{a_{0}}=2 \pi f$, we can express $k\left|\eta_{e}\right|$ in terms of the physical frequency observed today $f$

$$
k\left|\eta_{e}\right|=\frac{f}{f_{e}}
$$

where

$$
f_{e}=\frac{H_{e}}{2 \pi\left(1+z_{e q}\right)}\left(\frac{t_{e}}{t_{e q}}\right)^{1 / 2} \simeq 10^{9}\left(\frac{H_{e}}{10^{-4} M_{P}}\right)^{1 / 2} \mathrm{~Hz} .
$$

Here we have chosen the reference values for $H_{e}$ as $10^{-4} M_{P}$ and taken in order to reach to the numerical values in (56)

$$
\begin{aligned}
t_{e} & =\frac{1}{2 H_{e}}, \quad 1+z_{e q} \simeq 2.4 \times 10^{4} \Omega_{0} h_{0}^{2}, \\
t_{e q} & \simeq 4.1 \times 10^{10} \Omega_{0}^{-2} h_{0}^{-4} s \\
M_{p} & =1.22 \times 10^{19} \mathrm{GeV}=1.85 \times 10^{43} \mathrm{~Hz} .
\end{aligned}
$$

Thus, the resulting spectrum of the relic gravitational wave can be written as

$$
h_{0}^{2} \Omega_{g w}=1.2 \pi^{3} \times 10^{-14}\left(\frac{H_{e}}{10^{-4} M_{P}}\right)^{2}\left(\frac{f^{4}}{f_{e}^{4}}\right) N_{k} .
$$

Similarly, $k\left|\eta_{1}\right|$ can be expressed by

$$
k\left|\eta_{1}\right|=\frac{f}{f_{1}}
$$

where

$$
\begin{aligned}
f_{1} & =\frac{H_{1}}{2 \pi\left(1+z_{e q}\right)} e^{-\mathcal{N}_{1}}\left(\frac{t_{e}}{t_{e q}}\right)^{1 / 2} \\
& =e^{-\mathcal{N}_{1}} \frac{H_{1}}{H_{e}} f_{e}
\end{aligned}
$$

and $\mathcal{N}_{1}=\int_{t_{1}}^{t_{e}} H d t$. If we assume $H_{1} \simeq H_{e}$ during inflation, since $\mathcal{N}_{1} \gg 1$, we have always $f_{1} \ll f_{e}$.

The modes having frequencies with $f<f_{1}$ exit the horizon during the trans-Planckian regime and the modes with $f_{1}<f<f_{e}$ cross outside the horizon during the inflationary period in which the linear dispersion relations hold $\left(\left|\eta_{e}\right|<|\eta|<\left|\eta_{1}\right|\right)$. Since the characteristic frequency corresponding to the radiation-matter equal time $\left(\eta_{e q}\right)$ is $f_{e q} \simeq 10^{-16} \Omega_{0} h_{0}^{2} \mathrm{~Hz}$, the modes that have a frequency $f>f_{e q}$ today re-enter the horizon during the radiation 
dominated phase and the modes with $f<f_{e q}$ during the matter dominated phase. In this paper, we focus on the frequencies with $f>f_{e q}$.

Since it is only meaningful to consider $f \ll f_{e}$, we can approximated the produced number of particles as

$$
\frac{f^{4}}{f_{e}^{4}} N_{k} \simeq \frac{1}{4}+\frac{5}{4}\left|\mathcal{B}_{k}\right|^{2}-\operatorname{Re}\left(\mathcal{A}_{k} \mathcal{B}_{k}^{*}\right)
$$

For the usual standard inflation scenario with an adiabatic initial vacuum $\left(\mathcal{A}_{k}=1, \mathcal{B}_{k}=0\right)$, since $N_{k} \propto f^{-4}$, the relic gravitational wave shows the flat spectrum. Even if we consider the effect of the trans-Planckian regime, if $\eta_{1}$ is given by (17), as in (22) the relic spectrum of the gravitational wave is independent of $f$.

Taking into account of the ignorance of the transition process around at $\eta=\eta_{1}$ as in HL gravity (Sect. III), in this section we will leave $f_{1}$ as a free parameter.

If we define

$$
f_{c} \equiv\left(\frac{2}{\sqrt{b_{m}}}\right)^{1 / m} \frac{M}{H} f_{1}
$$

for $f \gg f_{1}$, using the asymptotic form of $H_{\nu}^{(2)}$, (23) , we obtain

$$
\begin{aligned}
\left|\mathcal{B}_{k}\right|^{2} & =\frac{1}{8}\left(\frac{f_{c}}{f}\right)^{m}\left[1+4\left(\frac{f}{f_{c}}\right)^{2 m}\right] \\
\operatorname{Re}\left(\mathcal{A}_{k} \mathcal{B}_{k}^{*}\right) & =\frac{1}{8}\left(\frac{f_{c}}{f}\right)^{m} \cos \left(\frac{2 f}{f_{1}}\right)\left[1-4\left(\frac{f}{f_{c}}\right)^{2 m}\right] .
\end{aligned}
$$

Hence, the spectrum for $f \gg f_{c}$ is

$$
h_{0}^{2} \Omega_{g w} \simeq 1.85 \times 10^{-13}\left(\frac{H_{e}}{10^{-4} M_{P}}\right)^{2}\left(\frac{f}{f_{c}}\right)^{m}\left[\frac{5}{4}+\cos \left(\frac{2 f}{f_{1}}\right)\right]
$$

and for $f \ll f_{c}$

$$
h_{0}^{2} \Omega_{g w} \simeq 4.6 \times 10^{-14}\left(\frac{H_{e}}{10^{-4} M_{P}}\right)^{2}\left(\frac{f_{c}}{f}\right)^{m}\left[\frac{5}{4}-\cos \left(\frac{2 f}{f_{1}}\right)\right] .
$$

As a consequence, for $f \gg f_{1}$, the shape of the spectrum changes at $f=f_{c}$. If we assume $f_{1} \propto f^{n}$ as in Sect. III, the spectrum grows as $f^{m(1-n)}$ if $f>f_{c}$ and decreases as $f^{-m(1-n)}$ if $f<f_{c} . m=0$ or $n=1$ gives a flat spectrum. Note that the expression (66) is, in fact, not exact form, because, for $f_{1} \ll f \ll f_{c}, z_{1} \propto\left(f / f_{c}\right)^{m} f / f_{1}$ and then the asymptotic form of the Hankel function (23) is not appropriate for this range. The exact result can be found numerically, which is shown in Fig. 1. 


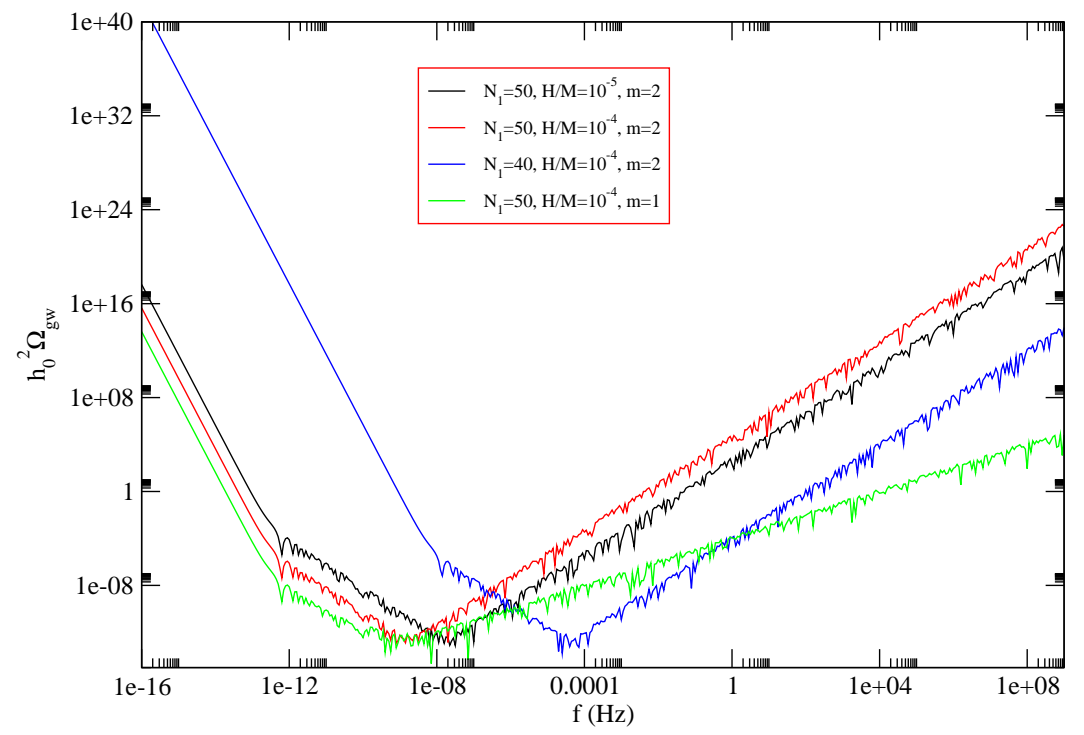

FIG. 1: $h_{0}^{2} \Omega_{g w}$ versus $f$ for different values of $\mathcal{N}_{1}, H / M$ and $m$

For $f \ll f_{1}$, the Hankel function is approximated as

$$
H_{\nu}^{(2)}(z) \simeq \frac{1}{i \sin \pi \nu}\left[\frac{e^{i \nu \pi}}{\Gamma(1+\nu)}\left(\frac{z}{2}\right)^{\nu}-\frac{1}{\Gamma(1-\nu)}\left(\frac{z}{2}\right)^{-\nu}\right], \quad \text { if } z \ll 1 .
$$

Since $z_{1} \ll 1, z^{-\nu}$ term is dominant over $z^{\nu}$ term and then we have

$$
\begin{aligned}
\left|\mathcal{B}_{k}\right|^{2} & \propto\left(\frac{M}{H}\right)^{\frac{3 m}{2(1+m)}}\left(\frac{f_{1}}{f}\right)^{\frac{9}{2}}, \\
\operatorname{Re}\left(\mathcal{A}_{a} \mathcal{B}_{k}^{*}\right) & \propto\left(\frac{M}{H}\right)^{\frac{3 m}{2(1+m)}}\left(\frac{f_{1}}{f}\right)^{\frac{9}{2}} .
\end{aligned}
$$

Using these coefficients, the spectrum becomes

$$
h_{0}^{2} \Omega_{g w} \propto\left(\frac{H_{e}}{10^{-4} M_{P}}\right)^{2}\left(\frac{M}{H}\right)^{\frac{3 m}{2(1+m)}}\left(\frac{f_{1}}{f}\right)^{\frac{9}{2}} .
$$

The spectrum for $f \ll f_{1}$ decreases $f^{-9(1-n) / 2}$ which is independent of $m$, where we have assumed $f_{1} \propto f^{n}$.

In Fig. 1, we plot the spectrum of the relic gravitational wave, $h_{0}^{2} \Omega_{g w}$, with the physical frequency observed today for different values of $\mathcal{N}_{1}, H / M$ and $m . m=2$ corresponds to HL gravity. The graph inflects at $f=f_{c}$ which is given in (62) and also bends at $f=f_{1}$. If we take $\mathcal{N}_{1}=50$ and $H / M=10^{-5}, f_{c} \simeq 10^{-8} \mathrm{~Hz}$. As expected from (65)), if $f>f_{c}$, the spectrum increases with $f^{m}$ as $f$ increases. And if $f_{1}<f<f_{c}$, it decreases for increasing $f$, but the slope is insensitive to the $m$ as explained in below (66). For $f<f_{1}$, the spectrum decreases with $f^{-9 / 2}$, independently of $m$, as $f$ increases. 


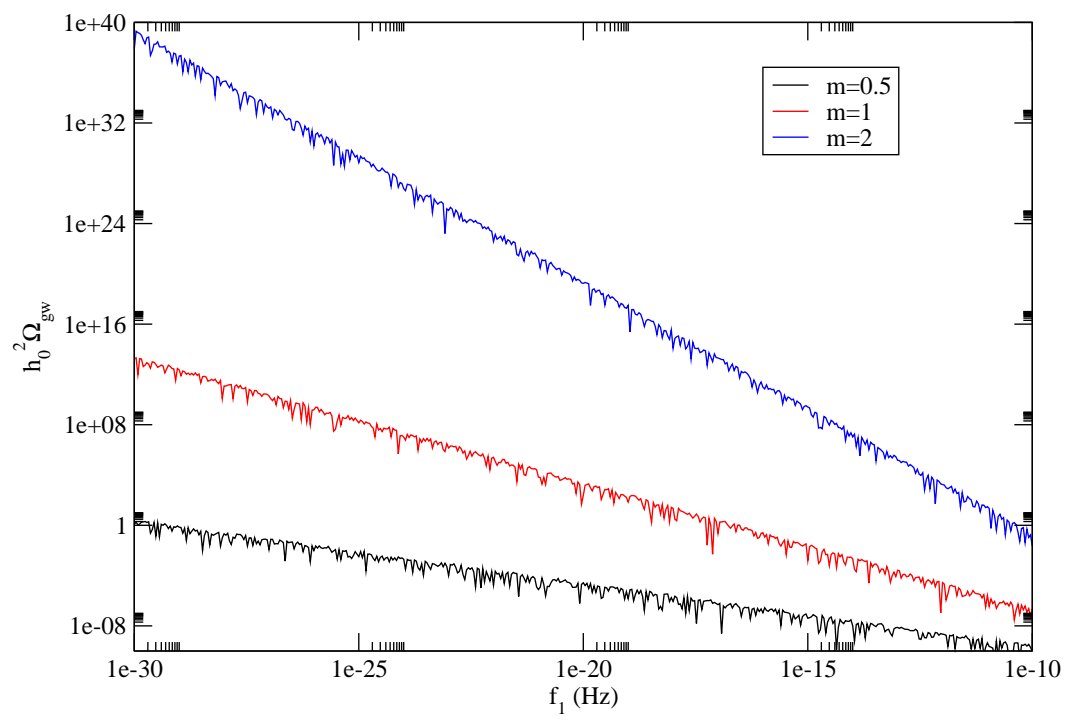

FIG. 2: $h_{0}^{2} \Omega_{g w}$ versus $f_{1}$ with $H / M=10^{-5}$ for $f=100 \mathrm{~Hz}$.

This figure seems to indicate the violation of the cosmological bounds from nucleosynthesis or CMB in the low frequency range [10] or the present ground-base gravitational wave detector (LIGO, VIRGO etc.) in the range $10 \mathrm{~Hz}<f<10^{3} \mathrm{~Hz}$. Even if $m=0$, since $h_{0}^{2} \Omega_{g w} \propto f^{-9 / 2}$ in the low frequency range, it might violate the cosmological bounds unless $\eta_{1}$ is constrained by (17). This implies that if $f_{1} \propto f^{n}$ where $n$ should be determined from the transition mechanism, we can expect $n \approx 1$ in order to satisfy the experimental and cosmological bounds.

In Fig. 2, we plot $h_{0}^{2} \Omega_{g w}$ as a function of $f_{1}$ at fixed $f=100 \mathrm{~Hz}$ which is the frequency range of the ground based gravitational wave detectors. The spectrum decreases for increasing $f_{1}$ independently of $m$. For a given $f_{1}$, as $m$ increases, the amplitude of the spectrum also increases. As a result, $f_{1}$ could be constrained from the experiment, if the gravitational waves are detected.

\section{SUMMARY AND DISCUSSION}

We have estimated the spectrum of the relic gravitational wave if the modified dispersion relations in the trans-Planckian regime is given by the Corley-Jacobson dispersion relations. The Corley-Jacobson type dispersion relations could be obtained from the recently suggested quantum gravity model called Hořava-Lifshitz gravity. Hořava-Lifshitz gravity was proposed 
as a model to be renormalizable quantum gravity theory in the UV region and Einstein gravity is expected to be recovered at the IR limit. $m=2$ in the Corley-Jacobson dispersion relations can be considered as HL gravity.

In obtaining the spectrum of the relic gravitational wave due to the effect of the transPlanckian physics, HL gravity phase is assumed to be followed by the inflationary phase in Einstein gravity and how to occur the transition seems to play an important role. As in many literatures dealing with the trans-Planckian effect, if the instantaneous transition time is chosen by the cut-off momentum, the resulting spectrum in the late time is scale invariant and leads to the correction term by $H / M$. Since it is, however, not clear how the transition from HL gravity in the UV regime to the IR limit i.e. Einstein gravity takes place, we leave $f_{1}$, the characteristic transition frequency, or $\eta_{1}$, the characteristic transition time, as a free parameter which is assumed to keep the information about the transition. We have found that in order to satisfy the experimental bounds and cosmological bounds from the nucleosynthesis or CMB, if we assume $f_{1} \propto f^{n}$ or $\eta_{1} \propto k^{-n}, n \approx 1$.

Although it is still controversial if HL gravity is a complete theory, it would be interesting to investigate whether HL gravity as like any other trans-Planckian physics can provide any detectable effects on the observations. And if HL gravity can provide the seeds for the large scale structure without inflation as well as solve the problems of the standard Big Bang cosmology, it would be also interesting to find some observational signature due to the tensor perturbations as well as the scalar perturbations in HL gravity without inflation [17].

\section{Acknowledgments}

The author would like to thank S. Mukohyama and G. Cristiano for useful comments.

[1] J. Martin and R. H. Brandenberger, Phys. Rev. D 63, 123501 (2001) arXiv:hep-th/0005209]; R. H. Brandenberger and J. Martin, Mod. Phys. Lett. A 16, 999 (2001) arXiv:astro-ph/0005432.

[2] S. Corley and T. Jacobson, Phys. Rev. D 54, 1568 (1996) arXiv:hep-th/9601073; S. Corley, Phys. Rev. D 57, 6280 (1998) arXiv:hep-th/9710075.

[3] P. Horava, Phys. Rev. D 79, 084008 (2009) arXiv:0901.3775 [hep-th]]. 
[4] P. Horava, JHEP 0903, 020 (2009) arXiv:0812.4287 [hep-th]].

[5] G. Calcagni, arXiv:0904.0829 [hep-th].

[6] E. Kiritsis and G. Kofinas, arXiv:0904.1334 [hep-th].

[7] S. Mukohyama, JCAP 0906, 001 (2009) [arXiv:0904.2190 [hep-th]]; Y. S. Piao, arXiv:0904.4117 [hep-th]; B. Chen, S. Pi and J. Z. Tang, arXiv:0905.2300 [hep-th]; R. G. Cai, B. Hu and H. B. Zhang, arXiv:0905.0255 [hep-th].

[8] M. Li and Y. Pang, arXiv:0905.2751 [hep-th]; D. Blas, O. Pujolas and S. Sibiryakov, arXiv:0906.3046 [hep-th]; C. Charmousis, G. Niz, A. Padilla and P. M. Saffin, arXiv:0905.2579 [hep-th].

[9] S. Mukohyama, arXiv:0905.3563 [hep-th]; S. Mukohyama, arXiv:0906.5069 [hep-th].

[10] M. Maggiore, Phys. Rept. 331, 283 (2000) arXiv:gr-qc/9909001.

[11] A. A. Starobinsky, Pisma Zh. Eksp. Teor. Fiz. 73, 415 (2001) [JETP Lett. 73, 371 (2001)] arXiv:astro-ph/0104043.

[12] M. Minamitsuji, arXiv:0905.3892 [astro-ph.CO].

[13] H. Lu, J. Mei and C. N. Pope, arXiv:0904.1595 [hep-th]; A. Kehagias and K. Sfetsos, arXiv:0905.0477 [hep-th]; R. A. Konoplya, arXiv:0905.1523 [hep-th]; E. O. Colgain and H. Yavartanoo, arXiv:0904.4357 [hep-th].

[14] C. Germani, A. Kehagias and K. Sfetsos, arXiv:0906.1201 [hep-th].

[15] T. Takahashi and J. Soda, arXiv:0904.0554 [hep-th]; M. Satoh, S. Kanno and J. Soda, Phys. Rev. D 77, 023526 (2008) arXiv:0706.3585 [astro-ph]].

[16] Y. f. Cai and Y. S. Piao, Phys. Lett. B 657, 1 (2007) arXiv:gr-qc/0701114.

[17] S. Koh, in preparation (2009). 